\title{
MiR-124 suppresses cell motility and adhesion by targeting talin 1 in prostate cancer cells
}

\author{
Wei Zhang ${ }^{1}$, Ye-qing Mao ${ }^{2}$, Hua Wang ${ }^{1}$, Wen-juan Yin ${ }^{3}$, Shao-xing Zhu ${ }^{1}$ and Wei-cheng Wang ${ }^{4}$
}

\begin{abstract}
Background: MicroRNA is a type of endogenous non-coding RNA implicated in various cellular processes, and has been intensely investigated in the field of cancer research for many years. Here, we investigated the functions and mechanisms of miR-124 in prostate cancer, which is a putative tumor suppressor reported in many carcinomas.

Methods: Using bioinformatics, talin 1 was indicated as a potential target of miR-124. We examined the expression levels of miR-124 and talin 1 in tissue specimens and cell lines. To explore the relationship between miR-124 and talin 1, miR-124 mimics, miR-124 inhibitors, and talin 1 small interfering RNA (siRNA) were transiently transfected into cancer cell lines, followed by analysis using luciferase reporter assays. Next, to investigate the functions of miR-124 in prostate cancer, we performed cell attachment, migration, and invasion assays. A rescue experiment was also conducted to demonstrate whether miR-124 suppressed cell adhesion and motility by targeting talin 1. Finally, we examined the related signaling pathways of miR-124 and talin 1.
\end{abstract}

Results: MiR-124 was down-regulated in prostate cancer specimens and cell lines, while talin 1 was over-expressed in prostate cancer specimens and cell lines. These results showed an inverse correlation of miR-124 and talin 1 expression. Similar to talin 1 siRNA, overexpression of miR-124 by transient transfection of mimics led to a significant decrease in talin 1 levels. Luciferase report assays showed that the seed sequence of the talin $13^{\prime}$-untranslated region was a target of miR-124. Functional investigations revealed anti-attachment, anti-migration, and invasion-promoting effects of miR-124 in prostate cancer cells. The rescue experiment confirmed that miR-124 exerted its biological functions by targeting talin 1. Finally, we found that miR-124 and talin 1 impaired cellular adhesion and motility through integrins and the focal adhesion kinase/Akt pathway.

Conclusions: Our study demonstrated biological roles and the related mechanism of miR-124 in prostate cancer. The results indicate that talin 1 is very likely a novel player in the anti-metastatic signaling network of miR-124. By down-regulation of talin 1, miR-124 impairs the adhesion, migration, and invasion of prostate cancer cells.

Keywords: MiR-124, Talin 1, Prostate cancer, Migration, Invasion, Adhesion, Integrins

\section{Background}

In 1949, Huggins et al. revealed the androgen-driven trait of prostate cancer. Subsequently, hormone therapies were developed to treat this deadly male malignancy. Although the survival rate has been extended in the past few decades, complications such as metastasis still lead to the deaths of nearly 30,000 men per year. Curative treatments for men with metastatic lesions remain elusive [1].

MicroRNAs (miRNAs) are a class of small (19-25 nt) non-coding RNAs that play important roles in gene

\footnotetext{
* Correspondence: herrmau@163.com

${ }^{2}$ Department of Urology, The First Affiliated Hospital of Zhejiang University,

79 Qinchun Road, Hangzhou, Zhejiang 310003, China

Full list of author information is available at the end of the article
}

regulation by partial or full complementary matching with the 3 '-untranslated region (UTR) of target mRNAs and triggering transcriptional or post-transcriptional suppression $[2,3]$. They are involved in numerous physiological functions such as cell differentiation, migration, proliferation, apoptosis, and senescence [4]. MiR-124, a putative tumor suppressor located in 8q12.3, is frequently found to be down-regulated in several human malignancies including bladder cancer, hepatocellular carcinoma, breast cancer, glioma, glioblastoma, cholangiocarcinoma, gastric cancer, osteosarcoma, ovarian cancer, and prostate cancer [5-17]. The biological effects of miR-124 in tumor cells are mainly negative regulation of cell proliferation, apoptosis, 
and especially metastasis. In particular, miR-124 is closely involved in tumor cell migration and invasion by targeting various molecules related to activation of the RhoA/ROCK pathway [6], matrix metalloproteinases (MMPs) [12,13,17], epithelial-mesenchymal transition $[9,15]$, and even epigenetic modification [11], which are pivotal events in tumor metastasis.

Here, we identified a mechanism of the anti-metastasis effect of miR-124 in prostate cancer. By directly targeting talin 1 , an integrin-associated cytoskeletal protein in focal adhesion formation, miR-124 suppresses the adhesion and motility of prostate cancer cells and down-regulates the focal adhesion kinase (FAK)/Akt pathway, suggesting the involvement of talin 1 in prostate cancer.

\section{Results}

Inverse correlation between talin 1 and miR-124 levels in prostate cancer

To investigate the expression of miR-124 in prostate cancer, its expression levels were examined quantitatively in 37 pairs of surgical specimens from prostate cancer patients by real-time PCR. In comparisons of the cancerous tissue and adjacent non-cancerous tissue in each pair, we found decreases of miR-124 expression at varying degrees in the cancerous tissues $(>50 \%$ reductions, $p<0.05$ ) (Figure 1A), which is consistent with previous reports $[9,13]$. Next, we examined the expression of talin 1 . The results showed that talin 1 was universally over-expressed in cancerous tissue compared with the adjacent tissue $(>2$ fold increases, $p<0.05$ ) (Figure 1A). The correlation analysis revealed an inverse relationship between miR-124 and talin 1 expression in these specimens (Figure 1B). Some studies have demonstrated that talin 1 is closely related to tumor metastasis $[18,19]$. Therefore, the surgical specimens were classified into a non-metastasis group (23 cases) and metastasis group (14 cases) according to the pathological results of lymph node biopsies. The metastasis group showed a modest increase in talin 1 expression compared with the non-metastasis group $(\sim 1.25$ fold, $p<$ 0.05 ) (Figure 1C). However, no differences in miR-124 expression were found between the two groups (data not shown).

Subsequently, miR-124 and talin 1 were examined and compared in a panel of prostate cancer cell lines and a normal prostate epithelial cell line, which showed similar results to the tissue specimens $(p<0.05)$ (Figure 1D-F).

These results not only confirmed up-regulation of talin 1 expression in prostate cancer, but also indicated an inverse correlation between miR-124 and talin 1 expression levels in prostate cancer, which implied a potential relationship.

\section{Talin 1 is a direct target of miR-124}

To investigate the potential link between talin 1 and miR-124, we searched for putative target genes using TargetScan (http://www.targetscan.org/) and focused on

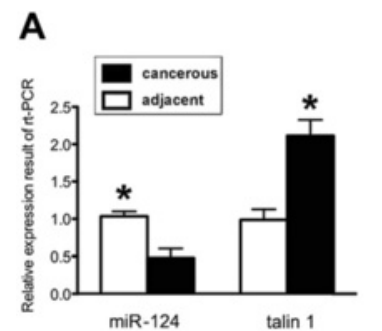

D

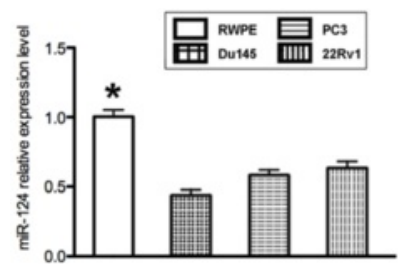

B

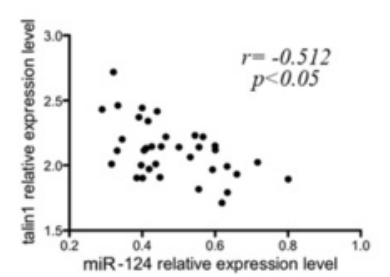

E

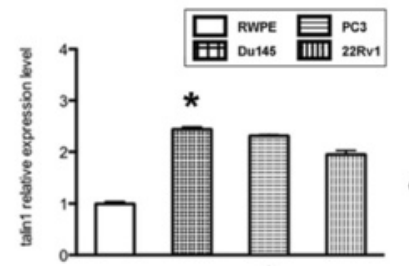

C

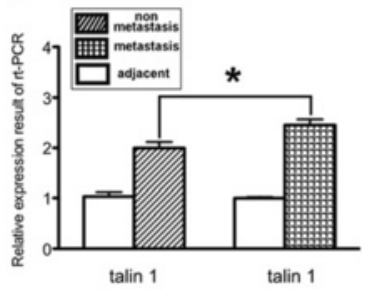

$\mathbf{F}$

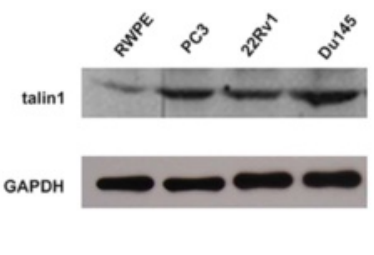

Figure 1 Expression levels of miR-124 and talin 1 in prostate cancer. (A) Quantification of miR-124 and talin 1 expression in surgical specimens from 37 prostate cancer patients by real-time PCR. The relative expression level of miR-124 in cancerous tissue was reduced by more than half of that in adjacent tissue, while the expression level of talin 1 in cancerous tissue was elevated by twice that in adjacent tissue. (B) Correlation analysis of miR-124 and talin 1 expression in the specimens demonstrated an inverse relationship ( $r=-0.512)$. (C) Comparison of talin 1 levels in the metastasis group (14 cases) and non-metastasis group (23 cases) revealed a relatively higher level of talin 1 in the metastasis group by approximately 1.25-fold. (D) Levels of miR-124 were examined in the prostate cancer cell lines, which showed a remarkably reduced level of miR-124 compared with the normal epithelial cell line. (E) Talin 1 levels were examined in prostate cancer cell lines, which showed remarkably elevated expression compared with the normal epithelial cell line. (F) Increased expression level of talin 1 in cell lines as shown by western blotting. Data represent the means from three independent experiments $\left({ }^{*} p<0.05\right)$. 
talin 1 because of its rank and associations with cell migration and invasion (Figure 2A). Considering the significantly low level of miR-124 in the Du145 cancer cell line, we overexpressed and knocked down miR-124 in Du145 cells by transfection of miR-124 mimics or inhibitors, respectively (Figure 2B). To investigate the effects of miR-124 on talin 1 expression, we examined talin 1 levels after manipulation of miR-124 expression. Ectopic expression of miR-124 substantially decreased the expression of talin 1, which resembled knockdown of talin 1 by small interfering RNA (siRNA), whereas suppression of miR-124 by the inhibitors resulted in remarkable up-regulation of talin 1 (Figure 2C). Therefore, miR-124 acts to negatively regulate the expression of talin 1 .

To confirm the relationship between miR-124 and talin 1, we performed a dual luciferase assay. HEK293T cells transfected with both the plasmid containing the target sequence in the talin $133^{\prime}$-UTR (pGL-TLN) and miR-124 mimics were defined as treatement group; the cells treated with the plasmid containing the mutated sequence (pGL-mut) and non-sense duplex sequence (NC RNA) were defined as controls. We found a specific interaction between exogenous miR-124 and the talin 13 '-UTR, which led to suppression of luciferase activity (Figure 2D). These results demonstrated posttranscriptional regulation of talin 1 by miR-124. Therefore, talin 1 may be the novel target of miR-124.

\section{MiR-124 suppresses the motility and adhesion of prostate} cancer cells in vitro

To functionally investigate the biological role of miR124 in prostate cancer, we conducted gain-of-function experiments. Considering the implication of talin 1 in cell motility and adhesion, we performed transwell and attachment assays. The results showed that miR-124 transfectants not only exhibited a significant reduction in attachment to fibronectin, an essential component of the extracellular matrix $(\mathrm{ECM})(p<0.05)$ (Figure $3 \mathrm{~A})$, but also an impairment in cell migration and invasion capabilities (Figure 3B). These effects resembled those in cells with talin 1 knockdown by siRNA $(p<0.05)$. The results indicated that miR-124 might suppress the motility and adhesion of prostate cancer cells through talin 1 .

Forced expression of talin 1 restores the miR-124induced inhibition of cellular migration and invasion

To confirm regulation of talin 1 by miR-124 in cellular attachment, migration, and invasion, we ectopically expressed talin 1 by transfection of pCDNA3.1(-) containing the talin

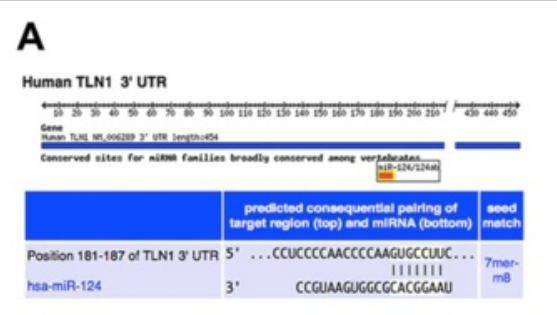

\section{B}

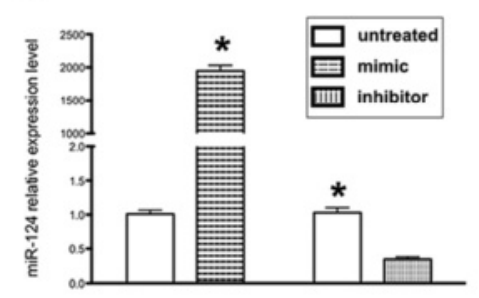

C

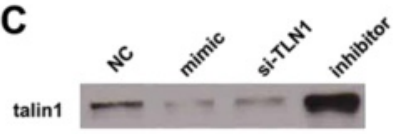

GAPDH
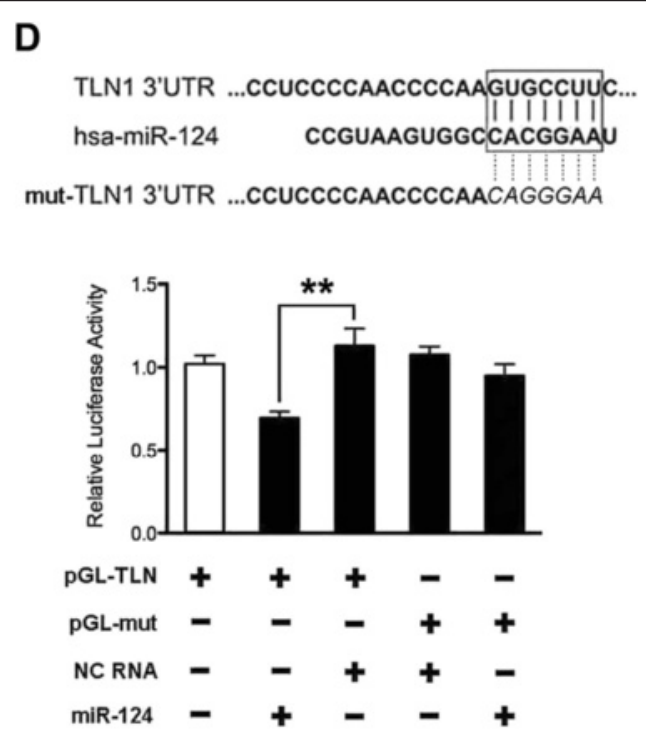

Figure 2 Talin 1 is a target of miR-124. (A) TargetScan revealed a putative interrelationship between talin 1 and miR-124. (B) To confirm transfection efficiencies, the level of miR-124 was examined after transfection of mimics or inhibitors. The miR-124 expression level was increased by approximately 2000-fold in cells transfected with mimics and decreased by $70 \%$ in cells transfected with inhibitors. (C) The expression level of talin 1 was obviously affected by manipulation of endogenous miR-124 in prostate cancer cells. (D) The dual luciferase reporter assay demonstrated regulation of talin 1 by miR-124. Relative light units were drastically suppressed by about $60 \%$ after transfection of pGL-TLN1 and mimics, implying an interaction between miR-124 and the seed sequence of the talin 1 gene. PGL-TLN1 denotes the reporter plasmid containing the seed sequence in the $3^{\prime}-U T R$ of the talin gene. pGL-mut denotes the reporter plasmid with a mutated seed sequence. Data represent the means from three independent experiments $\left({ }^{* *} p<0.01\right)$. 


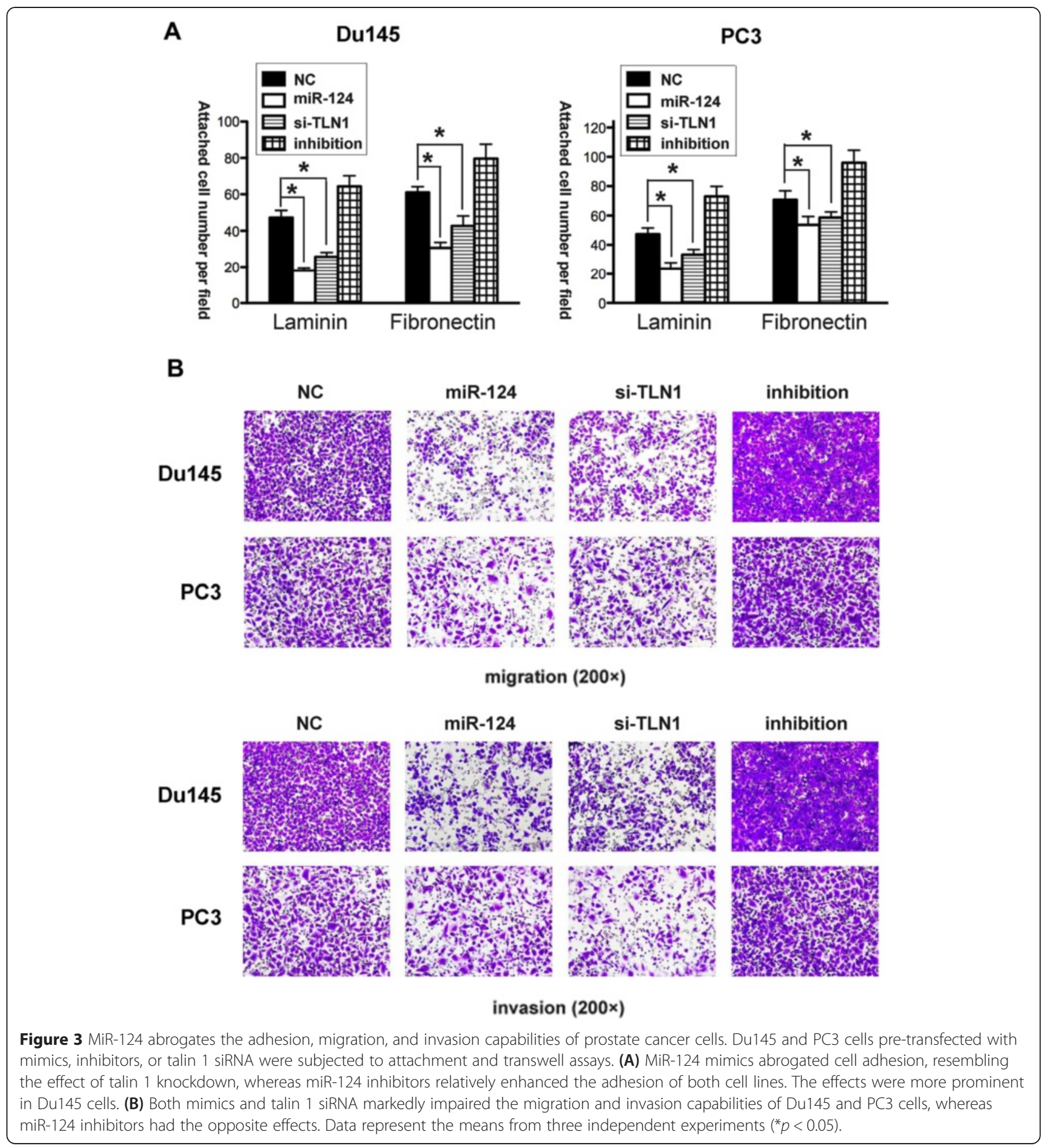

1-coding sequence together with miR-124 mimics into Du145 cells. The elevated expression level of talin 1 was remarkably mitigated by forced expression of miR-124 (Figure 4A, lanes 1 and 2). Conversely, the decreased level of talin 1 by miR-124 mimics was significantly restored by forced expression of talin 1 (Figure 4A, lanes
2 and 3). Co-transfection of NC RNA and a non-sense plasmid (p-CON) was used for the control (Figure 4A, lane 4). Next, an attachment assay (Figure 4B) and transwell assay (Figure 4C) clearly demonstrated that talin 1 overexpression restored, to a certain extent, the abrogated attachment and motility of cancer cells 

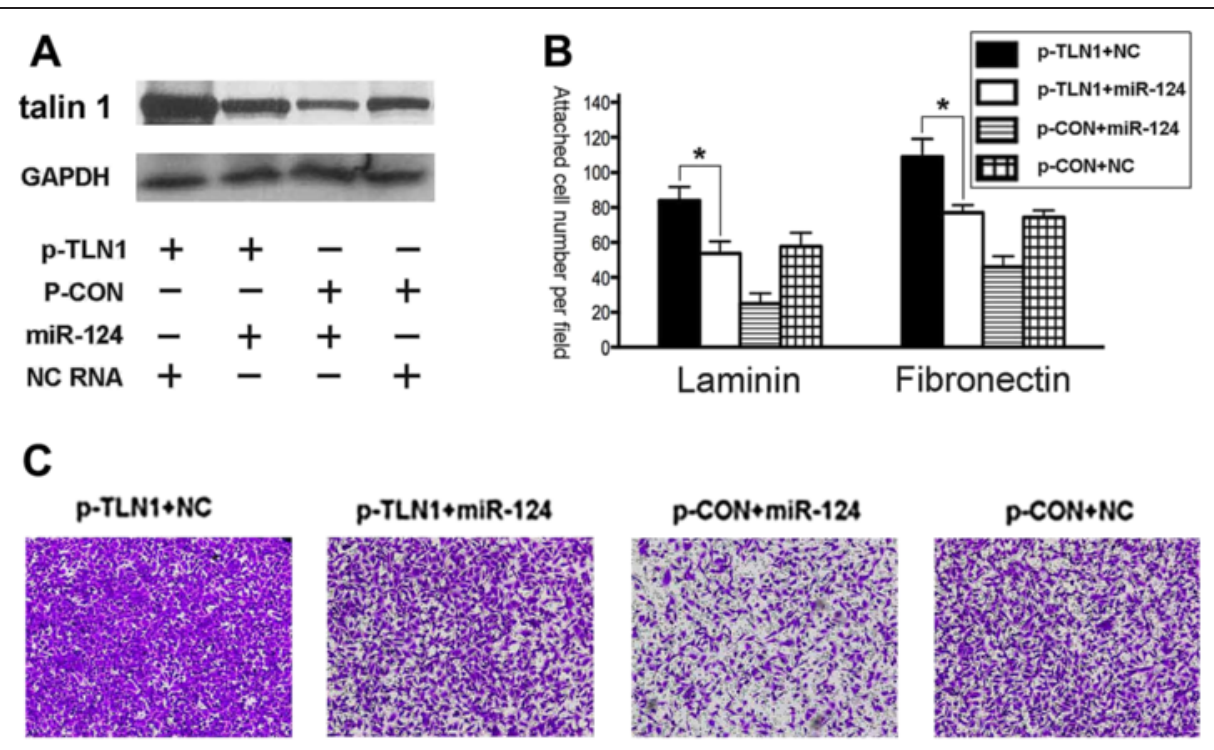

migration (200x)
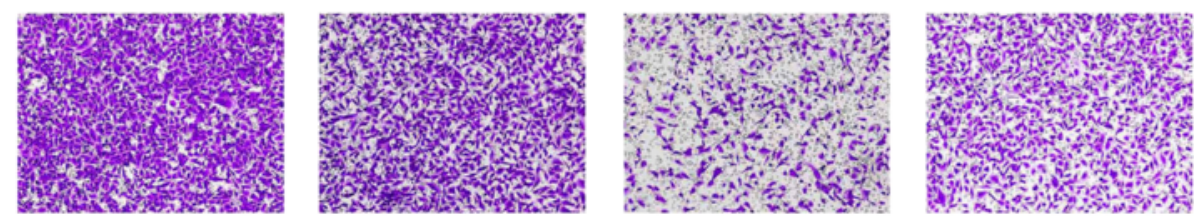

invasion (200x)

Figure 4 MiR-124 impairs cell adhesion and motility by targeting talin 1 directly. Du145 cells were co-transfected with miR-124 mimics or NC RNA and p-TLN or p-CON plasmids. (A) Western blot analysis of talin 1. The decreased level of talin 1 induced by miR-124 mimics could be restored by forced expression of talin 1. (B) Attachment and (C) Transwell assays demonstrated functional effects in cells after transfection. p-TLN denotes the expression plasmid containing the full-length coding sequence of talin 1. p-CON denotes the control plasmid containing the non-sense sequence. Data represent the means from three independent experiments $\left({ }^{*} p<0.05\right)$.

induced by miR-124 mimics. Evidently, miR-124 targeted talin 1 directly, resulting in suppression of adhesion and motility in prostate cancer cells.

\section{Mechanistic investigation of the suppressive effect of miR-124 via talin 1}

We confirmed that talin 1 is a direct and functional target of miR-124 in prostate cancer. Next, we investigated the mechanism of tumor suppression by miR-124 via talin 1 . Considering the varying levels of talin 1 among the prostate cancer cell lines, Du145 cells were selected for evaluation. Talin 1 acts as a mediator between integrins and the ECM through activation of integrins and recruitment of adaptor proteins. Recently, the FAK-src complex was identified as a pivotal partner in the interaction between talin 1 and the intracellular domains of integrins [20]. Aberrant activation of FAK-Src is common in tumorigenesis [21]. To better elucidate the suppressive role of miR-124 via talin 1 , an immunoprecipitation assay was performed with an anti-integrin $\beta 3$ antibody. The results showed a significant reduction in talin 1 binding to integrin $\beta 3$, as well as FAK and Src after transfection of miR-124 (Figure 5A). Next, the total and phosphorylated protein levels of FAK, AKT, and MAPK were examined by western blotting in Du145 cells. The levels of phosphorylated FAK, Akt, and MAPK were decreased by miR-124, suggesting inhibition of the FAK/Akt pathway (Figure 5B). Additionally, invasion-related effectors MMP2, MMP9, and E-cadherin were down-regulated in the miR-124 transfectants, which might account for the impaired invasive and migratory capabilities of prostate cancer cells with miR-124 overexpression (Figure 5C).

\section{Discussion}

As a type of adhesion receptor for ECM proteins, activated integrins promote tumor cell motility by forming focal adhesions [22]. Their activation requires adaptor proteins that facilitate interactions between integrins and actin fibers. Talin 1 is an important member of the adaptor protein family that plays an essential role in the activation of integrins. In mammals, there are two talin proteins encoded by TLN genes: ubiquitously expressed 


\section{A}
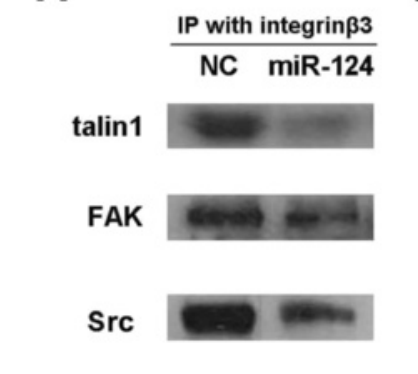

integrin $\beta 3$
B
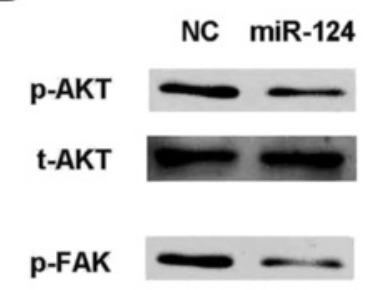

t-FAK

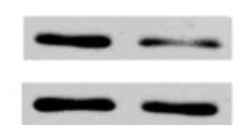

p-MAPK

t-MAPK
C

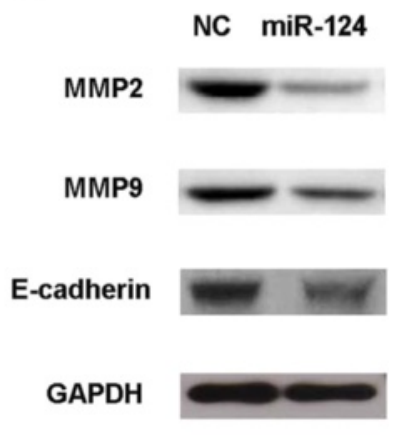

Figure 5 MiR-124 affects the downstream signaling pathway mediated by talin 1. DU-145 cells transfected with miR-124 mimics were subjected to immunoprecipitation using an anti-integrin $\beta 3$ antibody. Talin 1, FAK, and Src binding was detected by their corresponding antibodies. (A) The results showed decreases in the binding of talin 1, Src, and FAK to integrin $\beta 3$ after transfection of miR-124 mimics. (B) Levels of total and phosphorylated AKT (Ser473), FAK (Y397), and MAPK were down-regulated through suppression of the related signaling pathway by miR-124. (C) Levels of MMP2, MMP9, and E-cadherin proteins were decreased by transfection of miR-124 mimics.

talin 1 and tissue-specific talin 2 [23]. An abnormal increase in the expression level of talin 1 has been recently reported in prostate cancer. Sakamoto et al. examined talin 1 expression in prostate cancer cells. They found that the levels of talin 1 correlated well with the malignant degree of the lesions. A higher talin 1 level corresponded to poorer differentiation and higher Gleason scores in surgically resected specimens [19]. Similarly, in our study, a remarkably higher level of talin 1 was detected in cancerous tissue in comparison with the adjacent tissue, as well as in the metastasis group compared with the non-metastasis group. These findings confirmed an abnormal expression signature of talin 1 in human prostate cancer.

The $270-\mathrm{kDa}$ talin protein includes an N-terminal head domain, which is responsible for binding to the cytoplasmic tails of integrins, and a C-terminal flexible rod domain containing multiple binding sites for the Factin-binding protein vinculin and a second integrinbinding site $[24,25]$. Functionally, direct binding of talin to the intracellular domain of an integrin destabilizes the transmembrane complex of the integrin, resulting in remodeling of the extracellular compartments and activation [26,27]. Simultaneous binding to actin filaments forms stable linkages between the ECM and cytoskeleton, leading to integrin clustering, aggregates of ECM proteins [24], assembly of actin filaments, and the formation of focal adhesions. These events increase the migratory capability of cells.

To the best of our knowledge, only one study has focused on talin 1 as a molecular target of miRNA. Tang et al. found that miR-9 inhibits tumor cell proliferation, migration, and invasion by suppression of talin 1 in ovarian serous carcinoma [28]. Here, we employed miRNA to specifically suppress talin 1 levels through binding to the
3'UTR of talin 1. We searched for potential miRNAs related to talin 1 using an online bioinformatics tool, and screened miRNAs by luciferase reporter assays in prostate cancer cells. The results suggested that miR-124 targeted the talin 13 '-UTR by decreased levels of talin 1 in miR124 transfectants.

For function investigations, we mainly focused on inhibition of cell adhesion and motility by miR-124 and talin 1 . We simulated the extracellular environment of tumor cells by incubating the cells in fibronectin-coated dishes to examine their attachment capabilities. Similar to talin 1 knockdown, we found impaired adhesion of prostate cancer cells by over-expression of miR-124. In cell migration and invasion assays, significant reductions in the migration rates of cancer cells were observed in both miR-124 and talin 1 siRNA transfectants compared with the control group. Furthermore, a rescue experiment was performed to better illustrate the relationship between talin 1 and miR-124. It was clear that miR-124 counteracted the increase in talin 1 expression, resulting in mitigation of the enhanced cell adhesion and migration by talin 1 . Interestingly, enforced expression of talin 1 could not sufficiently restore the effects of miR-124 on cell migration and invasion. We attribute this observation to other metastasis-related pathways of miR-124. Recent studies have shown that miR-124 mitigates tumor cell migration by directly targeting ROCK and Rac-1, both of which are components of the RhoA-ROCK cascade that regulates actomyosin contractility $[6,12,29,30]$. Thus, it is very likely that the enhanced migration of talin 1-overexpressing cells was mitigated by the anti-migration effect of miR-124 to some extent.

FAK binds to the cytoplasmic domain of integrins in part by association with adaptor proteins talin and paxillin. In 
turn, activated FAK forms the FAK-Src complex via an interaction with Src, and further promotes the phosphorylation of many related substrates that participate in reorganization of the actin cytoskeleton as well as cell migration [20]. The FAK/Akt signaling cascade acts as an essential downstream effector of integrin-focal adhesion interactions [19]. To reveal the mechanism, we performed an immunoprecipitation assay using an anti-integrin $\beta 3$ antibody, and examined Akt, FAK, and MAPK, as well as their phosphorylated variants. The results showed that miR-124 and talin 1 might inactivate integrins and suppress the FAK/Akt pathway.

Src-FAK signaling has been recently reported to promote E-cadherin internalization, which facilitates tumor cell motility [31], inhibits the endocytic pathway, and activates MMP2 and MMP9 during cancer progression [32]. Accordingly, we identified suppression of effector proteins related to tumor invasion, MMP2, MMP9, and E-cadherin, in miR124-transfected tumor cells. These results might illustrate how miR-124 confers non-migratory and non-invasive phenotypes to prostate cancer cells by targeting talin 1 in an integrin-dependent manner.

\section{Conclusions}

Collectively, our study has shed light on the anti-migration and anti-invasion mechanisms of miR-124 in prostate cancer. Our preliminary experiments suggest that talin 1 is very likely a novel player in the anti-metastatic signaling network of miR-124, following ROCK, Rac-1, and transforming growth factor- $\alpha$. Considering the limitations of in vitro experiments, further in vivo investigations are needed to confirm these results.

\section{Methods}

\section{Clinical specimens}

From 2013, 37 patients diagnosed with prostate adenocarcinoma underwent radical prostatectomy at the Department of Urology, Zhejiang Cancer Hospital. Lymph node metastasis was determined according to pathological analysis of biopsies obtained by lymphadenectomy. For each specimen pair, an experienced pathologist discriminated the cancerous nodule from the adjacent non-tumor tissue.

\section{Cell culture and transient transfection}

Human prostate cancer cell lines PC3, Du145, and 22Rv1 , and the human prostate epithelial cell line RWPE were purchased from the Cell Bank of the Chinese Academy of Sciences (Shanghai, China). The human normal kidney cell line HEK293T was a kind gift by Dr Zhao An from the Central Laboratory of Zhejiang Cancer Hospital. All cells were maintained in RPMI-1640 medium (Invitrogen, Carlsbad, CA, USA) supplemented with 10\% fetal bovine serum (FBS; Gibco, Grand Island, NY, USA).
After transfection of miRNA and/or siRNA, cells were harvested, counted, and seeded into six-well plates (Costar, Corning, CA, USA). Lipofectamine $2000^{\text {ma }}$ reagent (Invitrogen) was employed to transfect siRNA (GenePharma, Shanghai, China) miR-124 mimics (RiboBio, Guangzhou, China), and miR-124 inhibitors (RiboBio, Guangzhou, China) into cells at 50, 100, and $200 \mathrm{nM}$, respectively. For mimics, NC RNA (the negative control), inhibitors, and siRNA, the duration of transfection was $48 \mathrm{~h}$. For co-transfection with plasmids, transfection was performed for $24 \mathrm{~h}$. The sequences were as follows $\left(5^{\prime}-3^{\prime}\right)$ : NC RNA, ACUACUGAGUGACAGUA GA; has-miR-124 [Pubmed Nucleotide: accession number: MI0000443], GGCAUUCACCUCGUGCCUUA; has-miR124 inhibitors, UAAGGCACGCGGUGAAUGCC; talin 1 siRNA, GAAGCCUCUUCUAUUUAAUGCAGAC.

\section{3'-UTR vector construction for luciferase reporter assays}

The talin 13 '-UTR fragment containing the seed sequence was amplified by PCR using cDNA from RWPE cells and the following primers: forward, 5 '-CGAGCTC CAGTCCCGCAGTACAT-3'; reverse, 5'-GCCGCGGT GGGGGAAGATAGTAT-3'. The amplified fragment was cloned downstream of the luciferase-coding sequence in a pmir-GLO expression vector (Promega, Wisconsin, USA) at the sites of Sal I and Sac I endonucleases (Takara, Dalian, China). The vector containing the seed sequence was called pGL-TLN1. A control vector containing a mutated sequence generated by a quickChange $^{\mathrm{Tw}}$ Site-directed Mutagenesis kit (Agilent Technologies, Santa Clara, CA, USA) was called pGLmut. HEK293T cells were transfected with $100 \mathrm{ng}$ pGLTLN1 + NC RNA, pGL-TLN1 + miR-124, pGL-mut + miR-124, and pGL-mut + NC RNA. After $24 \mathrm{~h}$, the cells were harvested and subjected to a Dual Luciferase Reporter Assay kit (Promega, Wisconsin, USA). The lysate was then analyzed by a bioluminescence detection system (Berthold Technologies, Bad Wildbad, Germany) to determine the relative light units.

\section{Transwell migration and invasion assays}

After transfection, $1 \times 10^{5}$ cells suspended in $200 \mu \mathrm{L}$ RPMI-1640 medium without FBS were added to the upper chamber of a Millicel transwell chamber $(8-\mu \mathrm{m}$ pore size, Millipore, Billerica, USA). For invasion assays, the filter membrane was coated with $30 \mu \mathrm{L}$ matrigel (BD Biosciences, Franklin Lakes, USA) diluted in RPMI1640 medium at a ratio of $1: 8$. A total of $600 \mu \mathrm{L}$ medium containing $10 \%$ FBS was added to the lower chamber. After incubation for $24 \mathrm{~h}$, the migrated/invaded cells were fixed with methanol, stained with $1 \%$ crystal violet, and counted under an inverted microscope. 


\section{Attachment assay}

After transfection, cells were collected, counted, and seeded in 24-well plates $\left(1 \times 10^{5}\right.$ cells per well) coated with fibronectin (BD Biosciences, Franklin Lakes, USA). After $1 \mathrm{~h}$ of incubation, the unattached cells were removed by washing with PBS three times. The remaining cells were fixed with methanol, stained with $1 \%$ crystal violet, and counted under an inverted microscope.

\section{Real-time RT-PCR}

To determine the relative expression levels of miR-124 and talin 1, total RNA and small RNA were extracted from cells using RNAiso Plus reagent and RNAiso for Small RNA reagent (Takara), respectively. cDNA of miRNA was then synthesized using a One Step PrimeScript ${ }^{\oplus}$ miRNA cDNA Synthesis Kit. Total cDNA was synthesized using a PrimeScript $^{\oplus}$ RT reagent Kit (Takara). Subsequently, real-time RT-PCR was performed with SYBR ${ }^{\odot}$ Premix Ex Taq $^{\text {Ts }}$ II (Takara) on an ABI 7500 Real-time PCR System (Applied Biosystems, Foster City, CA, USA). U6 and glyceraldehyde 3-phosphate dehydrogenase (GAPDH) genes were used as endogenous controls. The primers were as follows: miR124, 5' -TAAGGCACGCGGTGAATGCC-3'; U6, 5' -TGC GGGTGCTCGCTTCGGCAGC-3'; talin 1 forward, 5' -TG AGTCAGTGTGCCAAGAA-3' and reverse, 5' -TAGA TTCTGTACCACACT-3'; GAPDH forward, 5'-AAGG TGAAGGTCGGAGTCA-3' and reverse, 5' -GAAGAT GGTGATGGGATTT-3'. The relative expression levels of target genes were quantified by normalization to endogenous U6 or GAPDH expression levels, which was calculated as $2-{ }^{\Delta \Delta} \mathrm{C}(\mathrm{t})$.

\section{Immunoprecipitation}

Immunoprecipitation was performed with a Pierce Classic IP Kit (Thermo Scientific, Austin, TX, USA). DU-145 cells as well as NC RNA and miR-124 transfectants were lysed in RIPA buffer (Beyotime Biotech, Hangzhou, China). Total protein was extracted and quantified. Protein samples $(100 \mu \mathrm{g})$ were incubated overnight at $4^{\circ} \mathrm{C}$ with the antibody. After incubation of the lysates with Protein $\mathrm{G}$ Plus/Protein A agarose beads $(30 \mu \mathrm{L})$ for $1 \mathrm{~h}$ at $4^{\circ} \mathrm{C}$, the agarose was centrifuged in PBS and then subjected to SDS-polyacrylamide gel electrophoresis (PAGE). Finally, the proteins were transferred to $0.22-\mu \mathrm{m}$ nitrocellulose membranes (Abcam, Cambridge, MA, USA).

\section{Western blot analysis}

Total proteins in cells were extracted using RIPA buffer, quantified using a Pierce BCA Protein Assay Kit (Thermo Scientific, Austin, TX, USA), separated on 6\% SDS-PAGE gels, and then transferred onto nitrocellulose membranes. The membranes were blocked in 5\% dry skim milk for $1 \mathrm{~h}$ at room temperature, followed by incubation with each primary antibody overnight at $4^{\circ} \mathrm{C}$. Rabbit or mouse monoclonal antibodies against talin 1 were obtained from Abcam. Antibodies against FAK, Akt, phospho-FAK $\left(\mathrm{Y}^{397}\right)$, phospho-Akt $\left(\mathrm{S}^{473}\right)$, MAPK, and phospho-MAPK were purchased from Cell Signaling Technology (Beverly, MA, USA). Antibodies against integrin $\beta 3$, Src, MMP2, MMP9, E-cadherin, and GAPDH were from Epitomics (Burlingame, CA, USA). The membranes were then incubated with secondary antibodies and visualized using an EZ-ECL kit HRP (BioInd, Kibbutz Beit Haemek, Israel).

\section{Over-expression vector construction and rescue experiment}

To investigate the effect of talin 1 over-expression in cells, we designed a pair of primers (forward: $5^{\prime}$-CCGG ATCCATGGTTGCACTTTCACT-3'; reverse: 5'-CCG AATTCTAGAAGAGGCTTCTTT-3') to amplify the coding sequence of the talin 1 gene using CDNA from RWPE cells by PCR. The amplified sequence was then cloned into a pCDNA3.1+ expression vector (Invitrogen) using BamHI and EcoRI endonucleases (Takara). The vector containing the target sequence was called p-TLN1. A control vector with a mutated sequence generated by the quickChange $^{\mathrm{Tm}}$ Site-Directed Mutagenesis kit was called pCON. To directly examine the interaction between miR124 and talin 1 in prostate cancer cells, we performed a rescue experiment. The over-expression vector was transfected with Lipofectamine $2000^{\mathrm{rm}}$ reagent at $1 \mu \mathrm{g}$ per well. The cells were co-transfected with p-TLN1 + NC RNA, pTLN1 + miR-124, p-CON + miR-124, and p-CON + NC RNA for $24 \mathrm{~h}$ and then harvested for western blot and functional analyses.

\section{Statistical analysis}

The Student's t-test was performed using SPSS version 17.0 software for statistical analysis. A $p$-value of less than 0.05 was considered statistically significant. Data represent the means \pm standard deviation (standard deviation) of three independent measurements. The correlation analysis was performed and charted by the software Prism Graphpad version $5.0 \mathrm{c}$.

\section{Competing interests}

The authors declare that they have no competing interests.

\section{Authors' contributions}

WZ participated in all aspects of the experiments. HW carried out the molecular experiments. SXZ and WCWperformed the surgery and collected the surgical specimens. WJY performed pathological examinations of the surgical specimens. Drafting of the manuscript, rendering of the graphs and charts as well as the statistical analysis were carried out by YQM. All authors have read and approved the final manuscript.

\section{Acknowledgments}

Funding for the research was provided by 'Science and technology planning project of Zhejiang Province'(2014C33228). We thank the staff of the Medical Laboratory at Zhejiang Cancer Hospital for helpful advice. We thank Dr Zhao An who provided the HEK293T cells. We thank the staff of Edanz editing board for the great polishing. 


\section{Author details}

${ }^{1}$ Department of Urology, Zhejiang Cancer Hospital, 38 Guangji Road, Hangzhou, Zhejiang 310000, China. 'Department of Urology, The First Affiliated Hospital of Zhejiang University, 79 Qinchun Road, Hangzhou, Zhejiang 310003, China. '3 Department of Pathology, Zhejiang Cancer Hospital, 38 Guangji Road, Hangzhou, Zhejiang 310000, China. ${ }^{4}$ Medical equipment research institute of Zhejiang, 23 Huacheng Road, Hangzhou, Zhejiang 310000, China.

\section{Received: 21 November 2014 Accepted: 23 March 2015}

\section{Published online: 06 May 2015}

\section{References}

1. Siegel R, Ma J, Zou Z, Jemal A. Cancer statistics, 2014. CA Cancer J Clin. 2014;64(1):9-29.

2. Ambros $V$, Chen $X$. The regulation of genes and genomes by small RNAs. Development. 2007;134(9):1635-41.

3. Hutvagner G, Zamore PD. A microRNA in a multiple-turnover RNAi enzyme complex. Science. 2002;297(5589):2056-60.

4. Hwang HW, Mendell JT. MicroRNAs in cell proliferation, cell death, and tumorigenesis. Br J Cancer. 2007:96(Suppl):R40-4

5. Mucaj V, Lee SS, Skuli N, Giannoukos DN, Qiu B, Eisinger-Mathason TS, Nakazawa MS, Shay JE, Gopal PP, Venneti S et al: MicroRNA-124 expression counteracts pro-survival stress responses in glioblastoma. Oncogene 2014.

6. An L, Liu Y, Wu A, Guan Y. microRNA-124 inhibits migration and invasion by down-regulating ROCK1 in glioma. PLoS One. 2013;8(7), e69478.

7. Xie L, Zhang Z, Tan Z, He R, Zeng X, Xie Y, et al. MicroRNA-124 inhibits proliferation and induces apoptosis by directly repressing $E Z H 2$ in gastric cancer. Mol Cell Biochem. 2014;392(1-2):153-9.

8. Lee Y, Kim HJ, Park CK, Kim YG, Lee HJ, Kim JY, et al. MicroRNA-124 regulates osteoclast differentiation. Bone. 2013;56(2):383-9.

9. Qin W, Pan Y, Zheng X, Li D, Bu J, Xu C, et al. MicroRNA-124 regulates TGF-alpha-induced epithelial-mesenchymal transition in human prostate cancer cells. Int J Oncol. 2014;45(3):1225-31.

10. Lu Y, Yue X, Cui Y, Zhang J, Wang K. MicroRNA-124 suppresses growth of human hepatocellular carcinoma by targeting STAT3. Biochem Biophys Res Commun. 2013:441(4):873-9.

11. Li L, Luo J, Wang B, Wang D, Xie X, Yuan L, et al. Microrna-124 targets flotillin-1 to regulate proliferation and migration in breast cancer. Mol Cancer. 2013;12:163

12. $\mathrm{Xu} X, \mathrm{Li}$, Lin $Y$, Chen H, Hu Z, Mao Y, et al. MicroRNA-124-3p inhibits cell migration and invasion in bladder cancer cells by targeting ROCK1. J Transl Med. 2013;11:276.

13. Kang S, Zhao Y, Hu K, Xu C, Wang L, Liu J, et al. Cao F: miR-124 exhibits antiproliferative and antiaggressive effects on prostate cancer cells through PACE4 pathway. Prostate. 2014;74(11):1095-106.

14. Shi Z, Chen Q, Li C, Wang L, Qian X, Jiang C, et al. MiR-124 governs glioma growth and angiogenesis and enhances chemosensitivity by targeting R-Ras and N-Ras. Neuro Oncol. 2014;16(10):1341-53.

15. Zhang H, Wang Q. Zhao Q, di W: MiR-124 inhibits the migration and invasion of ovarian cancer cells by targeting SphK1. J Ovarian Res. 2013;6(1):84.

16. Chen Q, Lu G, Cai Y, Li Y, Xu R, Ke Y, et al. MiR-124-5p inhibits the growth of high-grade gliomas through posttranscriptional regulation of LAMB1. Neuro Oncol. 2014;16(5):637-51

17. Zeng B, Li Z, Chen R, Guo N, Zhou J, Zhou Q, et al. Epigenetic regulation of miR-124 by hepatitis $C$ virus core protein promotes migration and invasion of intrahepatic cholangiocarcinoma cells by targeting SMYD3. Febs Lett. 2012;586(19):3271-8.

18. Everley PA, Krijgsveld J, Zetter BR, Gygi SP. Quantitative cancer proteomics: stable isotope labeling with amino acids in cell culture (SILAC) as a tool for prostate cancer research. Mol Cell Proteomics. 2004:3(7):729-35.

19. Sakamoto S, McCann RO, Dhir R, Kyprianou N. Talin1 promotes tumor invasion and metastasis via focal adhesion signaling and anoikis resistance. Cancer Res. 2010;70(5):1885-95.

20. Mitra SK, Schlaepfer DD. Integrin-regulated FAK-Src signaling in normal and cancer cells. Curr Opin Cell Biol. 2006;18(5):516-23.

21. Kuphal $\mathrm{S}$, Bauer $\mathrm{R}$, Bosserhoff AK. Integrin signaling in malignant melanoma. Cancer Metastasis Rev. 2005;24(2):195-222.

22. Parsons JT, Horwitz AR, Schwartz MA. Cell adhesion: integrating cytoskeletal dynamics and cellular tension. Nat Rev Mol Cell Biol. 2010;11(9):633-43.
23. Monkley SJ, Pritchard CA, Critchley DR. Analysis of the mammalian talin2 gene TLN2. Biochem Biophys Res Commun. 2001;286(5):880-5.

24. Moser M, Legate KR, Zent R, Fassler R. The tail of integrins, talin, and kindlins. Science. 2009;324(5929):895-9.

25. Critchley DR, Gingras AR. Talin at a glance. J Cell Sci. 2008;121(Pt 9):1345-7.

26. Shattil SJ, Kim C, Ginsberg MH. The final steps of integrin activation: the end game. Nat Rev Mol Cell Biol. 2010;11(4):288-300.

27. Calderwood DA, Zent R, Grant R, Rees DJ, Hynes RO, Ginsberg MH. The Talin head domain binds to integrin beta subunit cytoplasmic tails and regulates integrin activation. J Biol Chem. 1999;274(40):28071-4.

28. Tang H, Yao L, Tao X, Yu Y, Chen M, Zhang R, et al. miR-9 functions as a tumor suppressor in ovarian serous carcinoma by targeting TLN1. Int J Mol Med. 2013;32(2):381-8.

29. Geng S, Zhang X, Chen J, Liu X, Zhang H, Xu X, et al. The tumor suppressor role of miR-124 in osteosarcoma. PLoS One. 2014;9(6), e91566.

30. Julian L, Olson MF. Rho-associated coiled-coil containing kinases (ROCK): structure, regulation, and functions. Small GTPases. 2014;5(2), e29846.

31. Benlimame $N$, He Q, Jie $S$, Xiao D, Xu YJ, Loignon M, et al. FAK signaling is critical for ErbB-2/ErbB-3 receptor cooperation for oncogenic transformation and invasion. J Cell Biol. 2005;171(3):505-16.

32. Wu X, Gan B, Yoo Y, Guan JL. FAK-mediated src phosphorylation of endophilin A2 inhibits endocytosis of MT1-MMP and promotes ECM degradation. Dev Cell. 2005;9(2):185-96.

\section{Submit your next manuscript to BioMed Central and take full advantage of:}

- Convenient online submission

- Thorough peer review

- No space constraints or color figure charges

- Immediate publication on acceptance

- Inclusion in PubMed, CAS, Scopus and Google Scholar

- Research which is freely available for redistribution 